\title{
Intracranial Hypotension After Severe Covid-19. Case Report and Literature Review.
}

\author{
Humberto Foyaca-Sibat ( $\square$ humbertofoyacasibat@gmail.com ) \\ Walter Sisulu University Faculty of Health Sciences https://orcid.org/0000-0002-6023-2394 \\ Lourdes de Fátima Ibañez Valdés \\ Walter Sisulu University - Mthatha Campus: Walter Sisulu University
}

\section{Case report}

Keywords: Coronavirus disease, 2019, SARS-CoV-2, intracranial hypotension, subdural hygroma, subdural effusion, cytokine storm, ACE-2, postCOVID-19 manifestations

Posted Date: February 19th, 2021

DOI: https://doi.org/10.21203/rs.3.rs-217250/v1

License: (c) This work is licensed under a Creative Commons Attribution 4.0 International License. Read Full License 


\section{Abstract}

Background: Despite the lack of clinical research done, new mutations of COVID-19 are travelling all over the world, and sooner or later, unexpected consequences going to be seen. In October 2020 new variant of SARV-CoV-2 (known as 20H/501.V2 or B1.351) has been found in our province (Eastern Cape), South Africa. This variant has multiple mutations in the spike protein, including K417N, E484K, N501Y. We did an extensive review of the medical literature (It is not a systematic review), looking for all publications regarding spontaneous intracranial hypotension, subdural effusions SARS-CoV-2/COVID-19.

Case Presentation: We also report a case of 46-years-old-female admitted severe COVID-19 in the intensive care unit; all performed investigations confirmed the severity of the infection caused by SARS-CoV-2. Past medical history proved a chronic headache, standard routine CSF analysis, and normal MRI of the brain three months before admission.

The patient recovered from the respiratory pathology and went home, but two weeks later, she developed a postural headache. We suspect spontaneous intracranial hypotension, and the CSF pressure $(5.5 \mathrm{~cm}$ of $\mathrm{H} 2 \mathrm{O})$ confirmed it. CT scan of the head showed bilateral frontal subdural effusion.

Conclusion: We did not find any published report related to COVID-19, subdural effusion, and intracranial hypotension. We elaborated a few comments and hypotheses to explain this process's probable pathophysiology.

\section{Introduction}

Next to the end of 2019, a new variant of coronaviruses affected many peoples causing viral pneumonia of unknown sources in Wuhan, belong to the Hubei province of central China. Later, this virus is named SARS-CoV-2 because some authors found similarities with the virus in 2003 caused the SARS [1]. From December 2019, the number of cases of 2019 coronavirus (COVID-19) infected subjects has been increasing exponentially up to date, accumulating more than 39 502,909 confirmed cases and 1 106,705 fatalities worldwide (on October 17, 2020) [2]. SARS-CoV-2 is a highly contagious virus transmitted from person-to-person through respiratory droplets, direct contact of mucous membrane with contaminated hands, or even vial faecal-oral contamination [3]. Despite lack of clinical research done, seem to be that a suspected new mutation of COVID-19 is travelling all over the world, and sooner or later, unexpected consequences going to be seen. Despite lack of clinical research done, seem to be that a suspected new mutation of COVID-19 is travelling all over the world, and sooner or later, unexpected consequences going to be seen. In October 2020 new variant of SARV-CoV-2 (known as 20H/501.V2 or B1.351) has been found in our province (Eastern Cape), South Africa. This variant has multiple mutations in the spike protein, including K417N, E484K, N501Y (www.virological.org).

According to the CDC in Atlanta (United States of America) and other authors, the most standard clinical features include cough, general malaise, fever, dyspnea, diarrhoea, chest pain, and muscle pain $[4,5]$. On the other hand, some neurological manifestations of COVID-19 include nonvascular headache, dry cough, sore throat, anosmia, ageusia, fatigue, myalgia, breathlessness and symptoms, and signs secondary ischemic and hemorrhagic stroke, cerebral sinus venous thrombosis, epileptic seizure, epilepsy, status epilepticus, Bell's palsy, Guillain-Barre-Syndrome including Miller Fisher variant, and encephalopathies among others [5-29]. There is no case report on intracranial hypotension in the medical literature despite around $88 \%$ of severe COVID-19 patients develop neurological complications with an associated lymphopenia, increased ferritin, and LDH level in blood [30]. Seems to be that the main entrance of the SARS-CoV-2 to the brain is via the systemic blood flow, mainly in severely infected patients. Another way of entry is passing the cribriform plate at the upper nasal fossa, causing hyposmia/anosmia, and then continuing via a retrograde axonal pathway to reach the brain [31]. The virus takes another path from mechanoreceptors and chemoreceptors in the infected lung tissue and then ascent vias synapse-connected route to the cardiorespiratory centre at the medulla oblongata [32] and from there most probable it disseminates to other areas of the brain and the cerebrospinal fluid (CSF). Some authors from Beijing Ditan Hospital (China) confirmed the SARSCOV-2 genome's presence in the CSF in March this year [33]. Other authors demonstrated the presence of COVID-19 in a case of necrotizing encephalopathy with damage to the blood-brain-barrier (BBB), leading to hemorrhagic encephalopathy [34]. Finally, other authors also confirmed SARS-CoV-2 in CSF in a child with encephalitis and endothelial vascular damage from virus invasion via angiotensin-converting enzyme II (ACE-2) receptors [35]

In a recent large study about the aggressivity of SARS = CoV-2, the authors found two significant virus types of modalities that they called L type and $S$ type being the first one the most aggressive compare with the second one [36]; these mutations can provide some impacts on the pathogenesis of the viruses. At the same time, other authors found a relationship between the severity of COVID-19 and chromosome 3p21.31 [37].

Several publications have been trying to explain the pathogenic mechanism behind the complications caused by COVID-19 [38-47]. One of the tools is the invasion of the brain via the receptor ACE-2 [38], and the structural and biophysical evidence 2019-nCoV S protein binds ACE2 (inhibited by ACE inhibitors) with higher affinity than does SARS-CoV S and suggesting that antibody cross-reactivity is limited amongst the two receptor-binding domains [39]. Recently, Want $\mathrm{K}$ et al. reported from their finding that SARS-CoV-2 invade host cells via a novel route of CD147- 
spike protein, which is bound to CD147 (a receptor on the host cells) [43]. Pia L. suggests that S protein-specific T cells in average persons may be cross-reactive clones developed after previous exposure to person endemic coronaviruses (cold) [44].

The severity of COVID-19 is directly proportional to the concentration level of tumour necrosis factor-alpha (TNF-a), macrophage inflammatory protein 1 alpha (MIP-1A), granulocyte colony-stimulating factor (GCSF), interferon gamma-induced protein 10 (IP-10), monocyte chemoattractant protein-1 (MCP-1), and inflammatory cytokines like interleukins (IL-2, IL-6, IL-7, IL-10) [47]. There is also an additional pathological process caused by programmed cell death protein 1 (PD-1), T-cell immunoglobulin mucin-3 (Tim-3), and natural killer group 2 A (NKG2A) at the beginning of the disease $[45,46]$. It is essential to highlight that NKG2A is an inhibitory component of the NKG2 group manifested on NK cells, natural killer T cells, and a subset of CD8 + T cells. The interactivity of NKG2A with histocompatibility antigen alpha chain E (HLA-E) can suppress NK cells and T cells [48]. T lymphocytes and NK cells are an expression of PD-1. These cells support the immune responses and promote self-tolerance, reducing $T$ cells' activity, stimulating regulatory $T$ cells [49]. T-cell immunoglobulin mucin-3 (Tim-3) (a co-inhibitory receptor) plays a crucial role in inhibiting $T$ helper 1 cells (Th1) responses and the expression of TNF- $\alpha$ and IFN-y [49].

Intracranial Hypotension ( $(\mathrm{H})$ is a clinical entity characterized by low CFS pressure $\leq 6 \mathrm{~cm}$ of $\mathrm{H} 2 \mathrm{O}$ measured at the lateral recumbency position without pillow by lumbar punction (LP) presenting clinical manifestations of postural headache, which is a positional headache which usually improves by lying in a recumbent position in 15-30 minutes [50], neck pain, nausea, vomiting, dizziness, and visual and hearing disturbances [51].In most cases, the leading cause is CSF leak at the setting level along the spine secondary to

1. The cause of the perineural cyst is the spontaneous dehiscence of dura matter.

2. Degenerative dural tears caused by the protrusion of calcified intervertebral disc.

3. Focal agenesia of dura matter (bare nerve root).

4. CSF-venous fistula [52]. IH can also be secondary to iatrogenic procedures, craniospinal injury, over-shutting (diversion devices).

Sometimes IH presents with unknown aetiology (Primary). This process is usually named spontaneous intracranial Hypotension (SIH) or craniospinal Hypotension.

$\mathrm{SIH}$ is more frequent in females around 30-50 years of age with a past medical history (PMH) of idiopathic intracranial hypertension, according to some authors [53]. The same authors also refer to PMH of clear rhinorrhea or otorrhea in case of traumatic or iatrogenic aetiology or even pituitary surgery through nasal fossa, sinus surgery basal skull fractures. Anecdotal SIH presentation in the comatose stage was reported to the literature [54]. Other less common exhibitions include medial sphenoid meningocele, Marfan syndrome, autosomal dominant polycystic kidney disease, idiopathic intracranial hypertension, Ehlers-Danlos syndrome (type II) [55].

Other conditions that can associate with SIH are subdural collections or hematomas. There is a predisposition of the bridging veins to broken and tear when they pass through the subarachnoid space, mainly in patients under anticoagulant therapy, causing chronic subdural hematoma (CSH) in around $5 \%-20 \%$ of the population $[56,57]$. Sometimes, the CSF volume loss may cause a compensatory enlargement of the subarachnoid/subdural space and occur a subdural hygroma (SHg) or a CSH if there is tearing of the engorged cortical veins even bilaterally [58]. Bilateral CSH also happens in SIH cases [58-60] or circumstances without SIH. In cases of underlying SIH, the CSH/SHg will not improve until SIH resolve.

Nevertheless, if the $\mathrm{CSH} / \mathrm{CHg}$ receives surgical treatment and disappears, then soon after, it may recur because of CSF underlying dynamics abnormalities [61]. Therefore, the primary treatment is to correct the leak instead of evacuating the subdural collection. This collection usually resolves following the appropriate SIH therapy [62]

The most relevant SIH features on MRI are diffuse pachymeningeal enhancement and "brain sag" signs, although these images are present in several clinical processes [63].

Finally, we are looking for two research questions: 1. What frequent is SIH in COVID-19 patients? 2. How COVID-19 can influence the pathogenesis of $\mathrm{SIH}$ ?

\section{Material And Method}

We search for publications about COVID-19 and associate subdural effusions and its pathophysiology and management to answer the two previous research questions using the procedure mentioned below and present our patient.

\section{Literature search strategy}


We included all studies (case reports, case series, and observational cohort studies) reported NCC/COVID-19/SE/ES/Ep during the initial search for all publications performed before September 10, 2020. We also reviewed the following databases for published studies: Medline EMBASE, Scopus online databases, Google Scholar, Science Direct, Scielo, LILACS, BIREME, and Cochrane library to identify articles evaluating COVID-19 and SE in NCC. All items about "spontaneous intracranial hypotension* OR subdural hygroma* OR subdural hematoma* OR neurological manifestations of COVID-19* OR coronavirus disease* OR cytokine storm* OR Neuro-COVID* OR pachymeningeal enhancement*OR subdural collection* OR acute epileptic seizures OR Neuro-COVID * OR seizure* OR COVID-19 OR unconsciousness" where * is the PubMed wildcard for every possible word beginning or ending. We did not consider other neurological manifestations beyond the current work scope. It was not a systemic review.

\section{Study and cohort selection}

We select all publications (case reports, case series, and observational cohort studies) reporting SIH, SH, SHg, and COVID-19 patients during the initial search. Later we progressively s excluded all duplicate studies. Those publications not meeting inclusion criteria because reported only COVID-19 separately, and SIH, SH, SHg not related to COVID-19, and those without an English translation.

\section{Results}

Between December 1 of 2019, and September 30, 2020, our literature search yielded 215 publications. After removing duplicate articles, we retained 59 unique records. Considering the title and abstracts, we discarded 21 journals, keeping 38 items, screening the full text, we selected 32 publications regarding COVID-19/Neurological complications. Finally, we found a total of 0 publications referring to COVID-19/SIH. From all groups, we did not find any published study about post-COVID-19/SIH/SH/SHg. (See FIGURE 1): Flow diagram of included studies for this review.

\section{Case Presentation}

A 46-years-old-female admitted at the Nelson Mandela Academic Hospital in June 2020 presented a four-day history of fever, dry cough, dyspnea, decreased taste and smelling sensation, and alternating day diarrhoea before admission. A fifth day from the beginning, she developed severe respiratory distress with severe hypoxemia, requiring a prolonged f stay in the intensive care unit (ICU), neuromuscular blockade, mechanical ventilation, and deep sedation.

Three months before the admission, she complained of a recurrent and severe non-vascular headache without focal neurological signs. CFS and CT scans of the brain were regular. Because the ELISA test came positive for cysticercosis, an MRI of the brain looking for intraventricular neurocysticercosis shows no abnormalities. She never smoked, consumed alcohol or recreational drugs.

On examination, moderate obesity is present with no noticeable body asymmetry. The patient had mild cyanosed mucous membranes but well hydrated and afebrile with a GCS 15/15; her motor examination revealed a standard power and no other neurological signs.

She was in respiratory distress with tachypnea of $30 \mathrm{breaths} /$ minute saturating at $84 \%$ on a $40 \%$ venture face mask. The rest of the vital signs showed a BP $125 / 85 \mathrm{mmHg}$, pulse $100 \mathrm{bpm}$. Hgt of 22mmol/I She had fine crepitation on both lower part of the chest and blood test on admission revealed: Hb: 13.1g/dl, White cell count $13.30 \times 109 / \mathrm{L}$, lymphocytes:741 $\mu \mathrm{L}(1,000$ to 4,800), Platelet count $365 \times 109 / \mathrm{L}$, CRP $23 \mathrm{H}$ $\mathrm{mg} / \mathrm{L}$. PCR confirmed SARS-CoV infection. Blood group A-positive, Na 141mmol/L, k 2.8mmol/L, Cl 118mmol/L, Urea $8.8 \mathrm{mmol} / \mathrm{L}, \mathrm{Creatinine}$ 122umol/L, level of D-dimer:0.85, Serum levels of interleukine- 6 were not available, Counts of CD4+ T cells: $310 / \mu \mathrm{L}$, ferritin $250 \mathrm{ng} / \mathrm{mL}$ (10 to 120 ), Creatine Kinase 612 units/L (30-135), procalcitonin: 0.94 ng/mL (0.10 - 0.49). Albumin: $7.8 \mathrm{~g} / \mathrm{dL}$ (3.4 to $5.4 \mathrm{~g} / \mathrm{dL}), \mathrm{CD} 4 \mathrm{count} 325 / \mu \mathrm{L}$.

When the patient arrived at ICU, she received high flow nasal oxygen (60\% at $15 \mathrm{~L} / \mathrm{min})$, Dexamethasone $8 \mathrm{mg}$ IV daily, Clexane $60 \mathrm{mg}$ SC 12 hourly, Ceftriaxone 1g IV daily, Azithromycin 500mg PO daily, Vitamin D 50000u PO weekly, Vitamin C 250mg PO 8hourly, Amlodipine 10mg orally daily, Ridaq $25 \mathrm{mg}$ orally daily and Intravenous fluids (1L Ringers lactate IV 8hourly).

A week after, the patient improved and was discharged from the hospital complaining of mild/moderate headaches from time to time and referred to the outpatient neurology clinic for follow-up. In the second week of discharge, she complained of a generalized headache that improves rapidity in the recumbent position and worsens around 15 minutes of assuming the upright position with complete recovery after 15 to 20 minutes in the supine position. This pain increased by coughing, laughing, and defecation if constipated. Then the patient returned to the neurology clinic where one of us (LdeFIV) did not find focal neurological signs, no meningeal signs, and fundoscopy was utterly ordinary. Still, the Valsalva manoeuvre exacerbates the headache remarkably. A lumbar punction did show an opening pressure of $5.5 \mathrm{~cm}$ of $\mathrm{H} 20$, and a CT scan of the brain confirmed a bilateral $\mathrm{SHg}$ in the frontoparietal region (Figure 2).

PCR denied SARS CoV-2 re-infection. The patient did not respond to oral analgesic received only by resting in flat position without pillow no less than 12 hours apart from generous caffeine intake (to stimulate CSF production), acetaminophen/ibuprofen, and increasing oral hydration. We also did a 48 hour Flat Test, which confirmed our diagnosis. The patient improved gradually, and it was no necessity to perform any invasive procedure, and never present any sign of rebound intracranial hypertension. 


\section{Comments And Final Remarks}

\section{First comments about results and flow diagram.}

The flow diagram (Figure 1) shows that we did not identify any publication regarding SIH/SD effusion/COVID-19 in our extensive research of the available medical literature. Therefore, we consider that this comorbidity or tardive neurological manifestation of COVID-19 is exceptionally uncommon. Later, we discuss possible pathophysiology for this finding and deliver our hypothesis for answering question two.

\section{Brief comments about the investigation done.}

Our second comment is related to an elevated white cell count, low lymphocyte count, elevated D-dimer level, and creatine kinase during her ICU admission suggested severe illness. Other authors also reported these findings when comparing these parameters among in and out ICU admissions [64]. We also found abnormal albumin levels, lactate dehydrogenase, and C-reactive protein probably associated with the lungs' acute injury during ICU admission. We could not check the status of angiotensin in plasma and viral load, which abnormalities have been reported by other authors as well [65]. Our patient belongs to blood group A, said in severe COVID-19 and respiratory failure [66, 67]. Findings of CD4 count below $400 / \mu \mathrm{L}$ associate with the severity of the SARS-CoV-2 infection have been reported too [46]. These authors also confirmed that T cell numbers related to IL-6, IL-10, and TNF-a concentration in the serum in COVID-19 patients were negative. When the total T cells account is below $800 / \mu \mathrm{L}$, those patients need urgent intervention, including a case with no apparent critical illness. Sooner or later, they will develop remarkable deterioration. It is essential to highlight that a notable of the lymphocytes' levels are part of the cases' characterization, presenting a severe COVID19 [45,46, 68]. Hypokalemia is the most relevant electrolyte imbalance in COVID-19 [47]. Apart from those mentioned before, another laboratory abnormality is relevant to determine the severity of the patient's condition and predictors of death, such as neutrophil/lymphocyte ratio, ferritin level, and hypophosphatemia hypofibrinogenemia, and platelet/lymphocyte ratio [47].

Other authors established that around 50\% of the critically ill COVID-19 patients present cytokine elevation, fever, high ferritin levels, and cytopenia $[69,70]$. It has known that SARS-CoV-2 causes an immunosuppressive effect on the body by exhausting and diminishing $T$ lymphocytes working with inhibitory receptors on natural killers and NKG2A, TIM-3, and PD-1 (T cells), as we mentioned before. Therefore, immunomodulators plus immunostimulant and anti-inflammatory activities can influence the severity of COVID-10 and minimize the risk of multiorgan failure and death [47]. Severe COVID-19, apart from causing neuronal and glial cells dysfunction, can develop other neurovascular complications and leptomeningeal injury [70].

Considering that SIH is a frequent consequence of CSF leak at any level along the neuroaxis, and this situation can alter the necessary equilibrium between the volume of arterial and venous blood, CFS, and brain parenchymal. That mechanism referred to the relationship between the intracranial components' pressure-volume inside the skull's no expansible compartments. It had been explained magisterially by Alexander Monro (1733-1817), a Scottish physician, and George Kellie (1720-1779). a Scottish general surgeon [71,72]. Their hypothesis combines the intracranial volume of the brain $(\sim 1400 \mathrm{~mL})$ with the total volume of the venous and arterial blood $(\sim 150 \mathrm{~mL})$ and the volume of the CSF $(\sim 150 \mathrm{~mL})$ to keep a dynamic equilibrium. When the volume of any component diminishes, the other two volumes increase to keep the intracranial volume average in adults ( 1700mL) [73]. One relevant mechanism controls the low-pressure venous system that displaces blood volume [72]. Suppose we apply the Monro-Kelly hypothesis to our patients. It is easier to understand the mechanism of low intracranial pressure in this case and why upright posture leads to the dilation of the nociceptive cerebral venous system explaining why orthostatic headache. On figure 3 , we list the most common causes of $\mathrm{SIH}$.

Some authors recently found evidence of neuronal degeneration, neuronophagia, mild oedema around nerve cells, and the small veins within the COVID civets' brain [74]. Based on this information, we can hypothesize about the subdural effusion mechanism caused by SARS-CoV-2 facilitated by a cytokine storm.

On the other hand, in critically ill COVID-19 patients, if possible, to see cerebral microbleeds (CM) damaging different areas of the brain, mainly the corpus callosum. This process's pathogenesis is still unknown, but the direct and indirect effect of SARS-CoV-2 on the endothelial tissue caused by the cytokine cascade is specific, apart from the hypoxemic development blood-brain-barrier (BBB) injury [75]. The best study to visualize CM is an MRI of the brain that we could not perform on our patients because of limited access to the machine due to our COVID protocol.

The pathogenesis of the brain lesions is related to astrocyte's disorder. This glial cell is the brain's primary homeostatic cell from one side. The other reactive astrocytes can release chemokines to activate specific receptors from invading microglia and macrophages, attracting them to the lesions. These astrocytes may modulate the immune response delivering TNF- $a$, IL-12, IFN- $y$, TGF- $\beta$, and IL-10 and controlling the pro-/antiinflammatory phenotype of macrophage [76].

The neuroinflammatory reactive astrocytes can stimulate naïve precursor T-cells and produce pro-inflammatory (Th1) or anti-inflammatory (Th2) phenotypes via pro-inflammatory cytokines (IFN- $\gamma$, TNF-a, IL-17) and anti-inflammatory cytokines (IL-10, TGF- $\beta$ ). 
Nevertheless, Th1 cells stimulate macrophages' activation and worsening the inflammatory reaction by releasing IL-2, IFN- $\gamma$, TNF, while Th2 cells cause inhibition of the macrophage's inflammatory activity by releasing IL-4, IL-5, IL-6, IL-10, and IL-13 through humoral immunity. The BBB integrity also depends on the astrocyte function by secreting VEGF, TGFa, bFGF, TNF-a, IL-1 $\beta$, IL-3, IL-6, Ang-1, BAFF, which support the endothelial cell control from one side of the barrier and the other by glial-derived neurotrophic factor. The nervous system's large glutamate sink is in the astrocytes, which control its function [76]. (See Figure 4).

Undoubling, astrocytes play an essential role in the mechanism of brain damage in human beings, depending on the predominance of cytokines released.

It has also known that many pathogenic agents cross the BBB by paracellular path, via transcytosis mechanism inside entering monocytes according to the Trojan Horse hypothesis or by the hijacking of $\beta$-adrenergic receptors [77]. The two BBB alterations are called non-disruptive and disruptive. The first one happens when there is molecular damage and its permeability if affected increasing or decreasing regulations of receptors and transportation across the barrier associate to astrocyte dysfunction, cytokine production and augmenting neuroinvasion of pathogen agents and the disruptive modality follow to anatomical modifications, including mitochondrial lesion, loss of tight junctions integrity, breakdown of glia limitans, degradation of the glycocalyx, increased vehicular traffic, re-induction of fenestrae, astrocytopathy, and apoptosis $[78,79]$. Astroglia-produced cytokines, [IL-1 $\beta$, IL-6, TNF-a], and prostaglandins mediate both BBB modalities [80].

Astroglia cells may release both anti/pro-inflammatory factors that can provoke and or control the neuroinflammatory brain process.

Among these anti-inflammatory agents' astrocytes released are:

· A.- cytokines and growth factors (IL-6, IL-10, IL-11, IL-19, IL-27, TGF- $\beta$, SHH).

B.- intracellular signalling factors (CRYAB; Gal9, STAT3, and A20)

C.- receptors (Dopamine D2 receptors, estrogenic receptor-a, glycoprotein 130.

D.- small intercellular effector molecules (MicroRNAs, retinoic acid: miR-181, miR-17-5p, and Dicer1).

Pro-inflammatory activity is listed here: (See Figure 4).

- A.- chemokines: monocyte chemoattractant protein-1 (MCP-1/CCL2), CCL5 (RANTES), CCL7, CCL8, CCL12, CXCL1, CXCL8 (IL-8), CXCL9, IFN- $\gamma-$ inducible protein-10 (IP-10/CXCL10), CXCL12, CXCL16.

- B.- cytokines and growth factors: IL1- $\beta$, IL-6, IL-11, IL-15, IL-17, TNF-a, BAFF, vascular endothelial growth factor (VEGF).

C.- intracellular signalling factors: NF-KB, SOCS3, Act1.

D.- small intercellular effector molecules: PGE and NO [81,82].

Without a doubt, SARS-CoV-2 cause brain inflammatory reaction as has been mentioned-before then; in that situation, astrocytes tend to upregulate the production of IL 1- $\beta$, IL-6, IL-11, IL-15, IL-17, TNF-a, BAFF, VEGF and develop remarkable delayed pro-inflammatory phenotype, even more than microglia $[83,84]$.

One of the most relevant pro-inflammatory activity in the pathogenesis of the septic process is provided by IL- $1 \beta$ inducing astroglia cells to produce thymidine phosphorylase (TYMP/endothelial cell growth factor 1, ECGF1). Vascular endothelial growth factor A (VEGF-A) contributing to the downregulation of TJ protein expression in BECs, leading to BBB breakdown [85]. At the same time, astroglia cells attenuate microglia activation releasing TGF- $\beta$ as well [86]. The before-mentioned activation of astrocytes with IL-1 $\beta$ supports the up-regulation of mRNA plus protein expression of IL-6 and TNF-a [87]. On the other hand, some authors found in a transgenic model that overexpression of IL- 6 (constitutive astrocytes) linked with a breakdown of the BBB, angiogenesis, increased expression of complement proteins, astrogliosis, and neurodegeneration $[88,89]$.

Other researchers also reported that SARS-CoV-2 infection increase levels of cytokines such as TNFa, interleukin (IL)-1 $\beta$, IL-6, IL-12, and INFY, a phenomenon known as "cytokine storm" (See Figure 4) [90-92].

Based on previous reports, SARS-CoV-2 cause neurodegeneration, demyelination, and cellular senescence, which accelerate the ageing process of the brain and increase neurodegenerative pathology [93-97] and we have hypothesized that damage persists beyond the acute phase of the disease because we could not identify a proper mechanism able to reverse these processes or accelerate the neurogenesis all over the brain.

Apart from the enunciated postulates about direct/indirect damage on the brain caused by SARS-CoV-2 through pro-inflammatory cytokines, chemokines, and growth factors; now we want to comment about other CNS damage seen in post-acute COVID-19 infection. In this regard, some 
authors confirmed enlargement of Rolandic operculum, insulas lobes, hippocampi, olfactory cortices, Heschl's gyrus, and cingulate gyrus by MRI images and reported from those prospective studies a general decline of mean diffusivity, axial and radial diffusivity accompanied by an increase of fractional anisotropy in the white matter of the right corona radiata, superior frontal-occipital fasciculus, and external capsule. Based on their findings, these authors suggest a disruption of the brain's micro-structural components and functionality in the recovery stages of COVID-19 [98].

To answer our second research question is necessary first to review the most acceptable pathophysiologic of ISH.

A dural tear is the cause of the syndrome in patients presenting connective tissue disorders like autosomal dominant polycystic kidney disease, Ehrler Danlos' syndrome (Type II), or Syndrome Marfan's Syndrome. In the case of dural ectasis leading to CSF leakage into the epidural or subdural space, this mechanism can explain why orthostatic headache and cranial nerves (V-VIII) disorders accompany this process [99]. Other conditions cause SIH like diabetic coma, hyperpnoea, uremia, dehydration, and even severe systemic illness [100]. Nevertheless, there are not COVID cases reported during the previous or current pandemic.

Based on observation of patients presenting SIH clinical manifestations with normal CSF pressure, we believe that reducing the CSF volume rather than a reduced CSF pressure is the primary pathophysiology of this syndrome but can be both processes together. Decrease CSF volume can be a consequence of the arachnoid membrane's rupture, leading to CSF leakage into the subdural space [101] how probably happened in our patient (See Figure 2). However, if this theory is correct, why are there not more COVID patients reported? Unfortunately, we have not a convincing answer to the previous question. Therefore, we can accept that this presentation may be comorbidity like a simple coincidence. Besides that, we still hypothesize that SARS-CoV-2 present in the CSF can cause damage to the arachnoids membrane until proven otherwise.

As we know, CSF's buoyancy plus pain-sensitive structures are the primary support of the brain. These structures are the meninges layers of the blood vessel (cerebral and cerebellar veins), some cranial nerves (5th, 9th, and 10th), and C1-C3 spinal nerves [102].

Then, when there is a stretching of these structures, some clinical manifestations of SIH are present. Considering the CSF's buoyancy, as mentioned earlier, the effect may diminish when its volume decreases, leading to the brain sag downwards, which causes headaches with worsening by an upright position like our patient. The same mechanism can explain postural headache in other similar patients or cases of reduced brain volume [103]. It is opportune to recall that SARS-CoV-2 can cause brain atrophy, as we point out before. Finally, headaches can be secondary to the intracranial blood vessels [104].

One of our patient's bedside tests with positive results was the Valsalva manoeuvre, which diminishes the venous return to the cardiac chambers and increases the venous volume intracranially, causing headaches even in a flat position. Some authors reported the presence of mononuclear pleocytosis, reticulocytes, elevated protein concentration, and xanthochromia in CSF of patients with SIH caused by diapedesis of protein and cells into the subarachnoid space [105], but we did not.

Here we briefly highlight some relevant signs seen on MRI in SIH cases. In this regard, Nadir Ali et al. [99] proposed a Mnemonic: SEEPS to remembering the five most relevant signs of SIH visible on MRI:

1. Sagging of brain or downward displacement of the brain.

2. Engorgement of venous structures

3. Enhancement of the pachymeninges

4. Pituitary hyperemia

5. Subdural fluid collection and the presence of extrathecal CSF.

Apart from reducing the angle between Galen vein and internal cerebral vein, collapsed superior ophthalmic vein, and spinal meningeal diverticula. However, the most characteristic MRI signs in SIH enhance the meninges, which is linear, non-nodular, and thick without the leptomeninges' involvement, which serves to differentiated SIH from meningitis [99].

Around $50 \%$ of patients with SIH have bilateral subdural effusion without any appreciable mass effect resulting in a remarkable meningeal enhancement caused by a compensatory effect of the cerebral veins dilatation secondary to huge CSF volume loss [106].

At this bibliographic research level, we found that leptomeningeal enhancement is present in COVID-19 even associated with the presence of oligoclonal bands $[107,108]$ and prominent subarachnoid spaces are quite common $(47 \%)$ around the optic nerves adding another interrogate about intracranial pressure in SARS-CoV-2 infected patients apart these volumetric and micro-structural pathological changes in the olfactory cortices and white matter of recovered COVID-19 cases as before mentioned [93].

Page $7 / 16$ 
One crucial issue that needs to be deeper investigate is the distribution of the ACE-2 all over the brain and its relationship with the affected brain regions considering that SARS-CoV-2 penetrate the nervous cells by attaching to ACE-2 through Spike glycoprotein that indicates more severe brain damage going to be present where the ACE-2 be more abundant. In these regards, the expression of ACE-2 is higher in substantia nigra and second place the spinal cord, followed by the hippocampus, lentiform nucleus, caudate nucleus, thalamus, limbic system, and lastly, frontal lobe cortex [109].

Probable the coincidence of an essential expression of SARS-CoV-2 in the frontal lobe leading to more concentration of SARS-CoV-2, the proximity to the main entrance of the virus into the brain (olfactory gyrus) as the first functional area in the nervous system invaded by SARS-CoV-2 [110] (anosmia) plus the microstructural and functional integrity changes at the recovery stages [93] and the damage caused by the pro-inflammatory cytokines over the meninges layers and arachnoid rupture [70] may explain the subdural effusion and SIH present in this patient. Almost finishing this writing, another question arises. Why is affected the frontal lobe and no others? (See Figure 2) It is probably because of the same explanation delivered before: more concentration of ACE-2 in the frontal lobe than the others, then more concentration of SARS-CoV-2 resulting in more regional pathology leading to frontal subdural effusion SIH. This figure (SARS-CoV-2/ CT scan) also has the intention to bring into your mind that coronavirus can affect the brain after respiratory syndrome recovery. Now we have not a clear idea how the mutation happened in the spike protein can cause damage in the brain cortex/subarachnoid space leading to subdural effusion. It is only a mental excise to elaborate hypotheses and reminders that other investigators need to confirm or deny.

\section{Limitations}

We acknowledge that our extensive review has several limitations. Firstly, many studies reviews are either case series or case reports, with only a few being observational cohort studies and no clinical trials performed. Most of these studies are considered relatively lower in quality with publication and reporting bias.

We report just a single case, and we could not confirm the effect caused by SARS-Cov-2 on the arachnoid membranes.

\section{Abbreviations}

ACE-2: Angio converting enzyme 3.

CNS: Central nervous system.

COVID-19: coronavirus disease, 2019.

$\mathrm{CSH}$ : Chronic subdural hematoma.

CT: computer tomography.

ECGF1 endothelial cell growth factor 1.

HCoV-229E: human coronavirus 229E.

HCoV-OC43: human coronavirus 0C43.

HCoV-NL63: human coronavirus NL63.

HCoV-HKU1: human coronavirus HKU1.

$\mathrm{IH}$ : Intracranial hypertension.

INFY: Interferon-gamma.

MERS-CoV: mild encephalitis /encephalopathy with a reversible splenial lesion and coronavirus.

SARS-CoV-2: Severe acute respiratory syndrome, coronavirus 2.

SHg: subdural hygroma.

SIH: Spontaneous intracranial hypotension.

TNF-a: Tumor necrotic factor alpha.

TYMP: thymidine phosphorylase. 
VEGF-A: Vascular endothelial growth factor-A.

\section{Declarations}

Ethical approval and consent to participate and publication: We obtained written informed consent to publish clinical details and patient's images. The Institutional Ethical committee did not consider this report for additional ethical approval.

Competing interest: None of the authors has any conflict of interest to disclose. The authors declare that they researched the absence of any commercial or financial relationships construed as a potential conflict of interest.

Funding: Both authors declare that they never received any financial support or personal collaboration that could have influenced the results reported in this paper.

Authors' contributions: Study concept and design: HFS and LFIV. Data collection: HFS and L de F IV. Analysis and interpretation of the references: LFIV. Drafting of the manuscript: LFIV, HFS. Revising the manuscript: HFS and LFIV. Supervision of research and manuscript writing process: HFS and LFIV. Both named authors meet the International Committee of Medical Journal Editors (ICMJE) criteria for authorship for this article, take responsibility for the work's integrity, and have given their approval for this version to be published.

Declaration of anonymity: Both authors certify that they did not reveal the names, initials, and other identity issues of this patient in this publication, and complete anonymity is guaranteed.

Availability of data and material: The data that support the findings of this study are available on reasonable request from the corresponding author.

Acknowledgement: Special thanks to Dr A Anwary from the Department of Radiology, NMACH. Mthatha, South Africa, for the report on images.

\section{References}

1. Zhou P, Yang X-L, Wang X-G. Pneumonia outbreak associated with a new coronavirus of probable bat origin. Nature. 2020;579(7798), 270273.

2. World Health Organization. Coronavirus disease 2019 (COVID-19) situation report. (2020). http://www.who.int/Docs/DefaultSource/Coronaviruse/Situation-Reports/20200402-Sitrep-73-Covid-19.Pdf?Sfvrsn=5ae25bc7_4\%20CDC-SRaF

3. Xiao F, Tang M, Zheng X, Liu Y, Li X, Shan H. Evidence for gastrointestinal infection of SARS-CoV-2. Gastroenterology. 2020;158(6), 18311833. e1833.

4. Centers for disease control and prevention. Symptoms of coronavirus. (2020). http://www.cdc.gov/coronavirus/2019-ncov/symptomstesting/symptoms.html

5. Guan W-J, Ni Z-Y, Hu Y. et al. Clinical characteristics of COVID-19coronavirus disease 2019 in China. N. Engl. J. Med. 2020;382(18), 17081720.

6. Nepal G, Rehrig JH, Shrestha GS, Shing YK, Yadav JK, Ojha R, Pokhrel G, Tu ZL, Ya-Huang D. Neurological manifestations of CoVID-19: a systematic review. Crit Care. 2020; 24: 421. doi: 10.1186/s13054-020-03121-z

7. Nepal G, Rehrig JH, Shrestha GS, Shing YK, Yadav JK, Ojha R, Pokhrel G, Tu ZL, Ya-Huang D. Neurological manifestations of COVID-19: a systematic review. Crit Care. 2020; 24: 421. doi: 10.1186/s13054-020-03121-z

8. Pinzon RT, Vincent Wijaya VO, Buana RB, Al Jody A, Nunsio PN. Neurologic Characteristics in Coronavirus Disease 2019 (COVID-19): A Systematic Review and Meta-AnalysisFront Neurol. 2020; 11: 565. doi: 10.3389/fneur.2020.00565.

9. Hao X, Zhou D, Li Z, et al. Severe psychological distress among epilepsy patients during the COVID-19 outbreak in southwest China. Epilepsia 2020. Doi:10.1111/epi.16544.

10. Moriguchi T, Harii N, Goto J, et al. The first case of meningitis/encephalitis associated with SARS-Coronavirus-2. Int J Infect Dis. 2020; $94: 55-$ 58. doi: 10.1016/j.jijid.2020.03.062.

11. Duong L, Xu P, Liu A. Meningoencephalitis without respiratory failure in a young female patient with COVID-19 infection in downtown Los Angeles, early April 2020. Brain Behav Immun.2020; 87:33. doi: 10.1016/j.bbi.2020.04.024.

12. Lu L, Xiong W, Liu D, et al. New-onset acute symptomatic seizure and risk factors in coronavirus disease 2019: a retrospective multicentre study. Epilepsia. 2020;61:e49-e53.

13. Fasano A, Cavalieri F, Canali E, Valzania F. First motor seizure as presenting symptom of SARS-CoV-2 infection. Neurol Sci.2020:1-3. doi: $10.1007 /$ s10072-020-04460-z.

Page 9/16 
14. Matilde Leonardi, Alessandro Padovani, Justin C. McArthur. Neurological manifestations associated with COVID-19: a review and a call for action. J Neurol. 2020 May 20: 1-4. doi: 10.1007/s00415-020-09896-z.

15. Vollono C, Rollo E, Romozzi M, et al. Focal status epilepticus as a unique clinical feature of COVID-19: a case report. Seizure. 2020; 78:109112. doi: 10.1016/j.seizure.2020.04.009.

16. Balloy G, Mahé PJ, Leclair-Visonneau L, et al. Non-lesional status epilepticus in a patient with coronavirus disease 2019. Clin Neurophysiol. 2020 doi: 10.1016/j.clinph.2020.05.005.

17. Somani S, Pati S, Gaston T, Chitlangia A, Agnihotri S. De Novo status epilepticus in patients with COVID-19. Ann Clin TransI Neurol. 2020 doi: 10.1002/acn3.51071

18. Daniele Orsucci, Elena Caldarazzo lenco, Gianpaolo Nocita, Alessandro Napolitano, Marco Vista. Neurological features of COVID-19 and their treatment: a review. Drugs Context. 2020; 9: 2020-5-1. doi: 10.7573/dec.2020-5-1.

19. Ghannam M, Alshaer Q, Al-Chalabi M, Zakarna L, Robertson J, Manousakis G. Neurological involvement of coronavirus disease 2019: a systematic review. J Neurol. 2020 June 19: 1-19. doi: 10.1007/s00415-020-09990-2.

20. Gelisse P, Rossetti AO, Genton P, et al. How to carry out and interpret EEG recordings in COVID-19 patients in ICU? Clin Neurophysiol. 2020;131(8): 2023-2030.

21. Mack Sheraton, Neha Deo, Rahul Kashyap, Salim Surani. A Review of Neurological Complications of COVID-19. Cureus. 2020 May; 12(5): e8192.doi: 10.7759/cureus.8192

22. Duong L, Xu P, Liu A. Meningoencephalitis without respiratory failure in a young female patient with COVID-19 infection in downtown Los Angeles, early April 2020. Brain Behav Immun. 2020; 87:33. doi: 10.1016/j.bbi.2020.04.024.

23. Pinzon RT, Vincent Wijaya VO, Buana RB, Al Jody A, Nunsio PN. Neurologic Characteristics in Coronavirus Disease 2019 (COVID-19): A Systematic Review and Meta-AnalysisFront Neurol. 2020; 11: 565. doi: 10.3389/fneur.2020.00565.

24. Xiong W, Liu D, et al. Acute symptomatic seizure and risk factors in coronavirus disease 2019: a retrospective multicenter study. Epilepsia. 2020;61: e49-e53. doi: 10.1111/epi.16524. [PMC free article] [PubMed] [CrossRef] [Google Scholar]

25. Somani S, Pati S, Gaston T, Chitlangia A, Agnihotri S. De Novo status epilepticus in patients with COVID-19. Ann Clin TransI Neurol. 2020 doi: 10.1002/acn3.5107

26. Hamid Reza Niazkar, Behdad Zibaee, Ali Nasimi, Narjes Bahri. The neurological manifestations of COVID-19: a review article. Neurol Sci. 2020 June 1: 1-5. doi: 10.1007/s10072-020-04486-3.

27. Sara Gómez Enjuto, Virgilio Hernando Requejo, José Lapeña Motilva, Gonzalo Ogando Durán, et al. Verapamil as a treatment for refractory status epilepticus secondary to PRES syndrome on a SARS-Cov-2 infected patient. Seizure. 2020 Aug; 80: 157-158.doi: 10.1016/j.seizure.2020.06.008

28. Mohammad A. Abdulsalam, Ahmad J. Abdulsalam, Diaa Shehab. Generalized status epilepticus as a manifestation of COVID-19. Acta Neurol Scand. 2020 July 20: 10.1111/ane.13321.doi: 10.1111/ane.13321

29. Michael O. Kinney, Francesco Brigo, Peter W. Kaplan. Optimizing status epilepticus care during the COVID-19 pandemic. Epilepsy Behav. 2020 Aug; 109: 107124. doi: 10.1016/j.yebeh.2020.107124

30. Mao L, Jin H, Wang M. et al. Neurologic manifestations of hospitalized patients with coronavirus disease 2019 in Wuhan, China. JAMA Neurol. 2020;77(6), 683-690.

31. Baig AM, Khaleeq A, Ali U, Syeda H. Evidence of the COVID-19 virus targeting the CNS: tissue distribution, host-virus interaction, and proposed neurotropic mechanisms. ACS Chem. Neurosci. 2020;11(7), 995-998.

32. Li YC, Bai WZ, Hashikawa T. The neuroinvasive potential of SARS-CoV2 may play a role in the respiratory failure of COVID-19 patients. J. Med. Virol. 92(6), 552-555 (2020).

33. Sun T, Guan J. Novel coronavirus and the central nervous system. Eur. J. Nerol. 2020. https://scholar.google.com/scholar_lookup? journal=Eur.+J.+Nerol.\&title=Novel+coronavirus+and+the+central+nervous+system\&author=T+Sun\&author=J+Guan\&publication_year=2020\&

34. Poyiadji N, Shahin G, Noujaim D, Stone M, Patel S, Griffith B. COVID-19-associated acute hemorrhagic necrotizing encephalopathy: CT and MRI features. Radiology. 2020;296(2): E119-E120.

35. Mcabee GN, Brosgol Y, Pavlakis S, Agha R, Gaffoor M. Encephalitis associated with COVID-19 infection in an 11-year-old child. Pediatr Neurol. 2020 Aug;109:94. doi: 10.1016/j.pediatrneurol.2020.04.013.

36. Tang X, Wu C, Li X. et al. On the origin and continuing evolution of SARS-CoV-2. Natl Sci. Rev. 2020;7(6), 1012-1023.

37. Ellinghaus D, Degenhardt F, Bujanda L. et al. Genomewide association study of severe Covid-19 with respiratory failure. N Engl J Med. 2020 June 17; NEJMoa 2020283. doi: 10.1056/NEJMoa2020283. 
38. Zou X, Chen K, Zou J, Han P, Hao J, Han Z. Single-cell RNA-seq data analysis on the receptor ACE 2 expression reveals the potential risk of different human organs vulnerable to 2019-nCoV infection. Front. Med. 2020;14, 185-192.

39. Wrapp D, Wang N, Corbett KS. et al. Cryo-EM structure of the 2019-nCoV spike in the prefusion conformation. Science. 2020;367(6483), 12601263.

40. Tikellis C, Thomas M. Angiotensin-converting enzyme 2 (ACE 2), is a crucial modulator of the renin-angiotensin system in health and disease. Int J Pept. 2012; 2012:256294. doi: 10.1155/2012/256294. Epub 2012 March 20.

41. Pal R, Bhansali A. COVID-19, diabetes mellitus, and ACE 2: the problem. Diabetes, Res. Clin. Pract. 2020;162, 108132.

42. Zhang S, Wang X, Zhang H. et al. The absence of coronavirus in expressed prostatic secretion in COVID-19 patients in Wuhan city. Reprod. Toxicol. 2020;96, 90-94.

43. Wang K, Chen W, Zhou Y-S. et al. SARS-CoV-2 invades host cells via a novel route: CD147-spike protein. Preprint from BioRxiv (2020).

44. Pia L. SARS-CoV-2-reactive T cells in patients and healthy donors. Nat Rev Immunol. 2020 Jun;20(6):353. doi: 10.1038/s41577-020-0333-2.

45. Zheng M, Gao Y, Wang G. et al. Functional exhaustion of antiviral lymphocytes in COVID-19 patients. Cell. Mol. Immunol. 2020;17(5), 533535.

46. Diao B, Wang C, Tan Y. et al. Reduction and functional exhaustion of T cells in patients with coronavirus disease 2019 (COVID-19). Front. Immunol. 2020;11, 827.

47. Elaheh Kordzadeh-Kermani, Hossein Khalili, Iman Karimzadeh. Pathogenesis, clinical manifestations, and complications of COVID-19. Future Microbiol. 2020 Aug : 10.2217/fmb-2020-0110.doi: 10.2217/fmb-2020-0110

48. Creelan BC, Antonia SJ. The NKG2A immune checkpoint-a new direction in cancer immunotherapy. Nat. Rev. Clin. Oncol. 16(5), 277-278 (2019).

49. Salmaninejad A, Valilou SF, Shabgah AG. et al. PD-1/PD-L1 pathway: basic biology and role in cancer immunotherapy. J. Cell. Physiol. 234(10), 16824-16837 (2019).

50. Schievink WI. Spinal cerebrospinal fluid leaks and intracranial hypotension. JAMA. 2006;295 (19): 2286-96. doi:10.1001/JAMA.295.19.2286.

51. Headache Classification Committee of the International Headache Society (IHS) The International Classification of Headache Disorders, 3rd edition. (2018) Cephalalgia. 38 (1): 1-211. doi:10.1177/0333102417738202-

52. Wouter I. Schievink, Franklin G. Moser, M. Marcel Maya. CSF-venous fistula in spontaneous intracranial hypotension. (2014) Neurology. 2014;83(5):472. doi:10.1212/WNL.0000000000000639.

53. 53. Frank Gaillard et al. Intracranial hypotension. Available at the URL: https://radiopaedia.org/articles/intracranial-hypotension-1.

54. Savoiardo M, Minati L, Farina L et-al. Spontaneous intracranial hypotension with deep brain swelling. Brain. 2007;130: 188493. doi:10.1093/brain/awm101- Pubmed citation

55. Morgan T. Spontaneous spinal cerebrospinal fluid leaks and intracranial hypotension. JAMA. 2007;328 (12): $2345-$ 52. doi:10.1001/JAMA.295.19.2286-

56. Luxon LM, Harrison MJ. Chronic subdural hematoma. Q J Med 1979; 48:43-53 pmid:482590.

57. Schievink WI, Maya MM, Pikul BK, et al. Spontaneous spinal cerebrospinal fluid leaks as the cause of subdural hematomas in elderly patients' anticoagulation: report of 3 cases. J Neurosurg 2010; 112:295-99 doi:10.3171/2008.10. JNS08428 pmid:19199465.

58. Huang YH, Yang KY, Lee TC, et al. Bilateral chronic subdural hematoma: what is the clinical significance? Int J Surg 2013; 11:544-48 doi: 10.1016/j.ijsu.2013.05.007 pmid:23707986.

59. Schievink WI, Maya MM, Moser FG, et al. Spectrum of subdural fluid collections in spontaneous intracranial hypotension. J Neurosurg 2005; 103:608-13 doi:10.3171/jns.2005.103.4.0608 pmid:16266041.

60. de Noronha RJ, Sharrack B, Hadjivassiliou M, et al. Subdural hematoma: a potentially serious consequence of spontaneous intracranial hypotension. J Neurol Neurosurg Psychiatry 2003; 74:752-55. doi:10.1136/ jnnp. 74.6.752 pmid:12754345.

61. Chen YC, Wang YF, Li JY, et al. Treatment and prognosis of subdural hematoma in patients with spontaneous intracranial hypotension. Cephalalgia, 2016;36:225-31. doi:10.1177/ 0333102415585095 pmid:25944817.

62. Rettenmaier LA, Park BJ, Holland MT, et al. Value of targeted epidural blood patch and management of subdural hematoma in spontaneous intracranial hypotension: case report and review of the literature. World Neurosurg 2017; 97:27-38 doi:10.1016/ j.wneu. 2016. 09.076 pmid:27693247.

63. K.M. Bond, J.C. Benson, J.K. Cutsforth-Gregory, D.K. Kim, F.E. Diehn, C.M. Carr. Spontaneous Intracranial Hypotension: Atypical Radiologic Appearances, Imaging Mimickers, and Clinical Look-Alikes. Amer J Neurorradiol July 2020, doi: https://doi.org/10.3174/ajnr.A6637

64. Wang D. Clinical characteristics of 138 hospitalized patients with 2019 novel coronavirus-infected pneumonia in Wuhan, China. JAMA 2020 Mar 17;323(11):1061-1069. doi: 10.1001/JAMA.2020.1585

65. Liu Y. Clinical and biochemical indexes from 2019-nCoV infected patients linked to viral loads and lung injury. Sci. China Life Sci. 2020.

Page $11 / 16$ 
66. Ellinghaus D, Degenhardt F, Bujanda L., et al. Genomewide association study of severe Covid-19 with respiratory failure. N. Engl. J. Med. (2020).

67. Tang N, Li D, Wang X, Sun Z. Abnormal coagulation parameters are associated with poor prognosis in patients with novel coronavirus pneumonia. J. Thromb. Haemost. 2020;18(4), 844-847.

68. Huang C, Wang Y, Li X. et al. Clinical features of patients infected with 2019 novel coronavirus in Wuhan, China. Lancet. 20202;395(10223), 497-506.

69. Fardet L, Galicier L, Lambotte O. et al. Development and validation of the HScore, a score for diagnosing reactive hemophagocytic syndrome. Arthritis Rheumatol. 2-14;66(9), 2613-2620.

70. Mehta P, Mcauley DF, Brown M. et al. COVID-19: consider cytokine storm syndromes and immunosuppression. Lancet 2020;395(10229), 1033.

71. Mokri B. The Monro-Kellie hypothesis: applications in CSF volume depletion. Neurology. 2001;56 (12): 1746-8. Neurology (full text)- doi:10.1212/WNL.56.12.1746-

72. Hickey JV. Clinical Practice of Neurological and Neurosurgical Nursing. Lippincott Williams \& Wilkins. (2011) ISBN:1451161018. Read it at Google Books.

73. Rangel-Castilla L, Rangel-Castillo L, Gopinath S et-al. Management of intracranial hypertension. Neurol Clin. 2008;26 (2): 521-41, x. doi:10.1016/j.ncl.2008.02.003.

74. Y. Xiao, Q. Meng, X. Yin, Y. Guan, Y. Liu, C. Li, M. Wang, G. Liu, T. Tong, L.-F. Wang, X. Kong, D. Wu.Pathological Changes in Masked Palm Civets Experimentally Infected by Severe Acute Respiratory Syndrome (SARS) Coronavirus. J Comp Pathol. 2008 May; $138(4)$ : $171-179$. doi: 10.1016/j.jcpa.2007.12.005.

75. Aikaterini Fitsiori, Deborah Pugin, Camille Thieffry, Patrice Lalive, Maria Isabel Vargas. Unusual Microbleeds in Brain MRI of Covid-19 Patients. J Neuroimaging. 2020 July 8: 10.1111/Jon.12755.doi: 10.1111/Jon.12755.

76. Tatyana Shulyatnikova, Alexei Verkhratsky. Astroglia in Sepsis Associated Encephalopathy. Neurochem Res. 2020; 45(1): 83-99. doi: 10.1007/s11064-019-02743-2.

77. Combes V, Guillemin GJ, Chan-Ling T, Hunt NH, Grau GE. The crossroads of neuroinflammation in infectious disorders: endothelial cells and astrocytes. Trends Parasitol. 2012; 28:311-319. doi: 10.1016/j.pt.2012.05.008.

78. Candelario-Jalil E, et al. Cyclooxygenase inhibition limits blood-brain barrier disruption following intracerebral injection of tumour necrosis factor-alpha in the rat. J Pharmacol Exp Ther. 2007; 323:488-498. doi: 10.1124/jpet.107.127035.

79. Minami T, Okazaki J, Kawabata A, Kawaki H, Okazaki Y, et al. Roles of nitric oxide and prostaglandins in the increased permeability of the Blood-Brain Barrier caused by lipopolysaccharide. Environ Toxicol Pharmacol. 1998; 5:35-41.

80. Varatharaj A, Galea I. The blood-brain barrier in systemic inflammation. Brain Behav Immun. 2017;60:1-12. doi: 10.1016/j.bbi.2016.03.010.

81. Sofroniew MV. Astrocyte barriers to neurotoxic inflammation. Nat Rev Neurosci. 2015; 16:249-263. doi: 10.1038/nrn3898.

82. Dong Y, Benveniste EN. Immune function of astrocytes. Glia. 2001; 36:180-190.

83. Bellaver B, et al. Systemic inflammation as a driver of brain injury: the astrocyte as an emerging player. Mol Neurobiol. 2018; 55:2685-2695. doi: 10.1007/s12035-017-0526-2.

84. Norden DM, Trojanowski PJ, Villanueva E, Navarro E, Godbout JP. Sequential activation of microglia and astrocyte cytokine expression precedes increased lba-1 or GFAP immunoreactivity following systemic immune challenge. Glia. 2016; 64:300-316. doi: 10.1002/glia.22930.

85. Chapouly C, et al. Astrocytic TYMP and VEGFA drive blood-brain barrier opening in inflammatory central nervous system lesions. Brain. 2015; 138:1548-1567. doi: 10.1093/brain/awv077.

86. Vincent VA, Tilders FJ, Van Dam AM. Inhibition of endotoxin-induced nitric oxide synthase production in microglial cells by the presence of astroglial cells: a role for transforming growth factor-beta. Glia. 1997; 19:190-198.

87. Lee SC, Liu W, Dickson DW, Brosnan CF, Berman JW. Cytokine production by human fetal microglia and astrocytes. Differential induction by lipopolysaccharide and IL-1 beta. J Immunol. 1993; 150:2659-2667.

88. Barnum SR, Jones JL, Muller-Ladner U, Samimi A, Campbell IL. Regular complement C3 gene expression in the CNS of transgenic mice with astrocyte-targeted interleukin-6 expression. Glia. 1996; 18:107-117. doi: 10.1002/(SICI)1098-1136(199610)18.

89. Campbell IL, Abraham CR, Masliah E, Kemper P, Inglis JD, Oldstone MB, Mucke L. Neurologic disease induced in transgenic mice by cerebral overexpression of interleukin 6. Proc Natl Acad Sci USA. 1993; 90:10061-10065.

90. Huang C, Wang Y, Li X, et al. Clinical features of patients infected with 2019 novel coronavirus in Wuhan, China. Lancet. 2020;395(10223):497-506. doi: 10.1016/S0140-6736(20)30183-5.

91. Huang K-J, Su I-J, Theron M, et al. An interferon-y-related cytokine storm in SARS patients. J Med Virol. 2005;75(2):185-194. doi: 10.1002/jmv.20255. 
92. Nicholls JM, Poon LL, Lee KC, et al. Lung pathology of the fatal severe acute respiratory syndrome. Lancet. 2003;361(9371):1773-1778. doi: 10.1016/s0140-6736(03)13413-7.

93. Yiping Lu, Xuanxuan Li, Daying Geng, Nan Mei, Pu-Yeh Wu, Chu-Chung Huang, Tianye Jia, Yajing Zhao, Dongdong Wang, Anling Xiao, Bo Yin. Cerebral Micro-Structural Changes in COVID-19 Patients - An MRI-based 3-month Follow-up Study. E Clinical Medicine. 2020 Aug; 25 : 100484.doi: 10.1016/j.eclinm.2020.100484.

94. Desforges M, Le Coupanec A, Stodola JK, Meessen-Pinard M, Talbot PJ. Human coronaviruses: Viral and cellular factors involved in neuroinvasiveness and neuropathogenesis. Virus Res. 2014; 194:145-158.

95. Lane TE, Hosking MP. The pathogenesis of murine coronavirus infection of the central nervous system. Crit Rev Immunol. $2010 ; 30: 119-130$.

96. Baz-Martínez M, Da Silva-Álvarez S, Rodríguez E. Cell senescence is an antiviral defense mechanism. Sci Rep. 2016; 6:1-11.

97. Hascup ER, Hascup KN. Does SARS-CoV-2 infection cause chronic neurological complications? GeroScience. 2020 doi: 10.1007/s11357-02000207-y.

98. Jasti M, Nalleballe K, Dandu V, Onteddu S. A review of pathophysiology and neuropsychiatric manifestations of COVID-19. J Neurol. 2020 doi: 10.1007/s00415-020-09950-w.

99. NadirAli, SyedFarhan, ArshadMirza, Aqueel Hussain Pabaney, Rameez-ul-Hassan. Pathophysiology and management of Spontaneous Intracranial Hypotension - A Review. J Pak Med Ass. 2012;62 (1): 23-31.

100. Bruera O, Bonamico L, Giglio JA, Sinay V, Leston JA, Figuerola ML. Intracranial hypotension: the nonspecific nature of MRI findings. Headache 2000; 40: 848-52.

101. Gaukroger PB, Brownridge P. Epidural Blood Patch in the treatment of spontaneous low CSF pressure headache. Pain 1987; 29 : 119-22.

102. Mokri B, Atkinson JL, Dodick DW, Miller GM, Piepgras DG. Absent pachymeningeal gadolinium enhancement on cranial MRI despite symptomatic CSF leak. Neurology 1999; 53: 402-4.

103. Messori A, Simonetti BF, Regnicolo L, Di Bella P, Logullo F, Salvolini U. Spontaneous Intracranial Hypotension: The Value of brain measurements in diagnosis MRI. Neuroradiology 2001; 43: 453-61.

104. Paldino M, Mogilne A, Tenner M. Intracranial Hypotension Syndrome: A comprehensive review. Neurosurg Focus 2003; 15 : ECP2.

105. Pannullo SC, Reich JB, Krol G, Deck MD, Posner JB. MRI changes in intracranial hypotension. Neurology 1993; $43: 919-26$.

106. Schievink WI, Maya MM, Moser FG, Tourje J. Spectrum of subdural fluid collections in spontaneous intracranial hypotension. J Neurosurg 2005; 103: 608-13.

107. Helms J, Kremer S, Merdji H, et al. Neurologic Features in Severe SARS-CoV-2 Infection. New England Journal of Medicine. 2020;382(23):2268-2270.

108. Kremer S, Lersy F, de Sèze J, et al. Brain MRI Findings in Severe COVID-19: A Retrospective Observational Study. Radiology. $2020 ; 202222$.

109. Chen R, Wang K, Yu J, Chen Z, Wen C, Xu Z. The spatial and cell-type distribution of SARS-CoV-2 receptor ACE2 in humans and mice brains. bioRxiv2020;2020.04.07.030650.

110. Netland J, Meyerholz DK, Moore S, Cassell M, Perlman S. Severe Acute Respiratory Syndrome Coronavirus Infection Causes Neuronal Death in the Absence of Encephalitis in Mice Transgenic for Human ACE2. J Virol. 2008; 82:7264-7275.

\section{Figures}




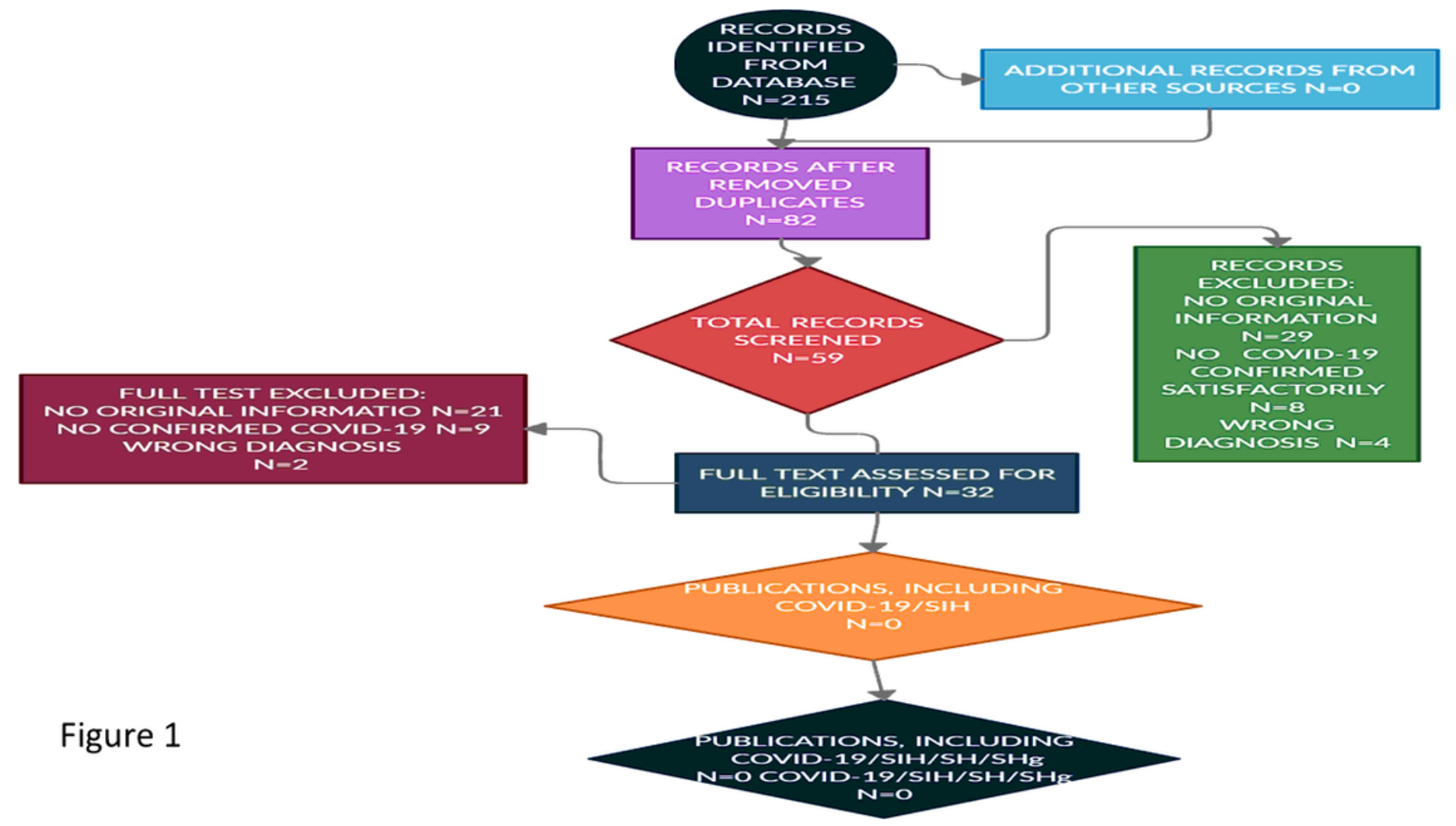

Figure 1

Flow diagram of publications searched in the medical literature.

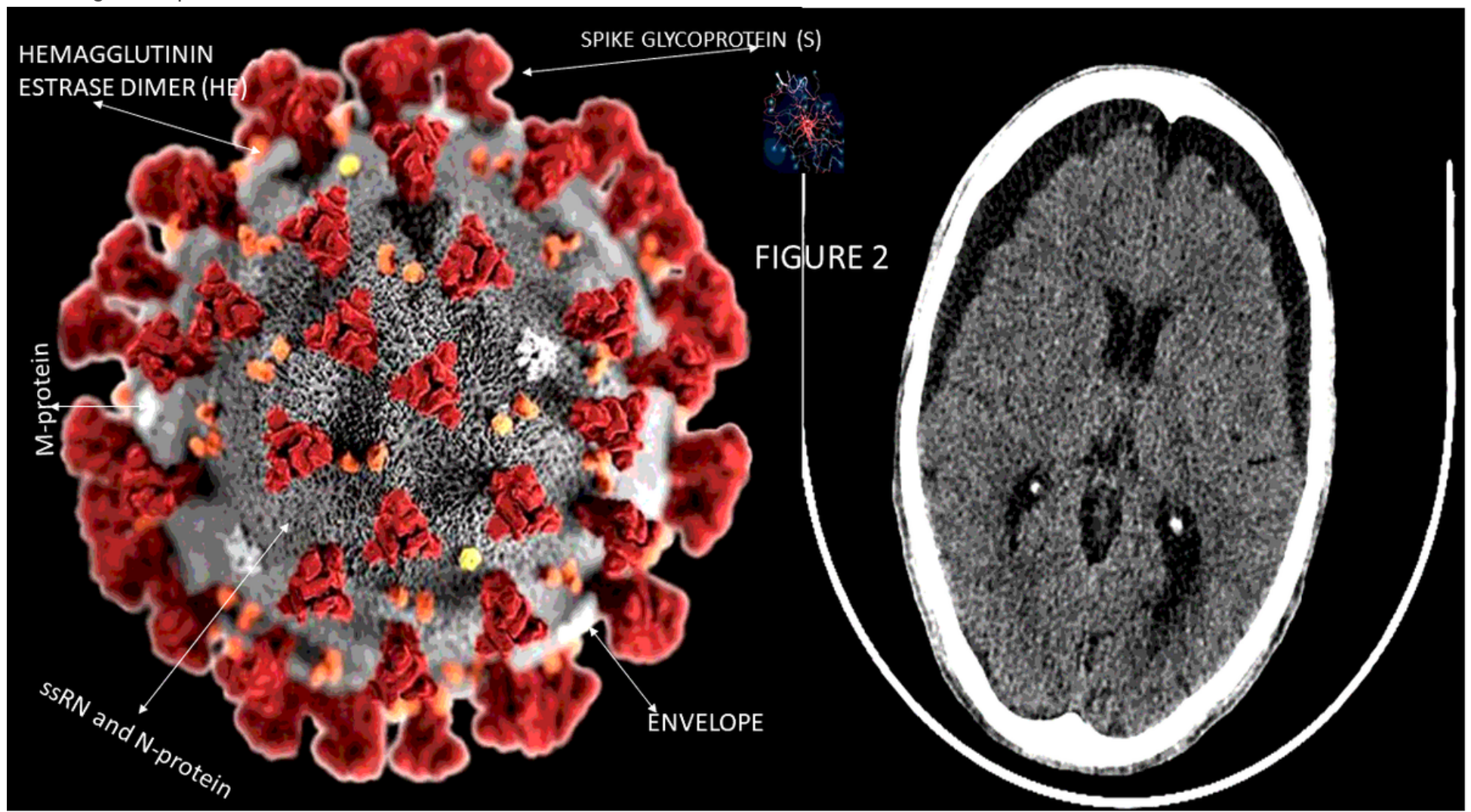

Figure 2 
(Left): Gross components of coronavirus 2. Right: CT scan of the brain shows a bilateral frontoparietal subdural effusion more prominent in the frontal lobe.

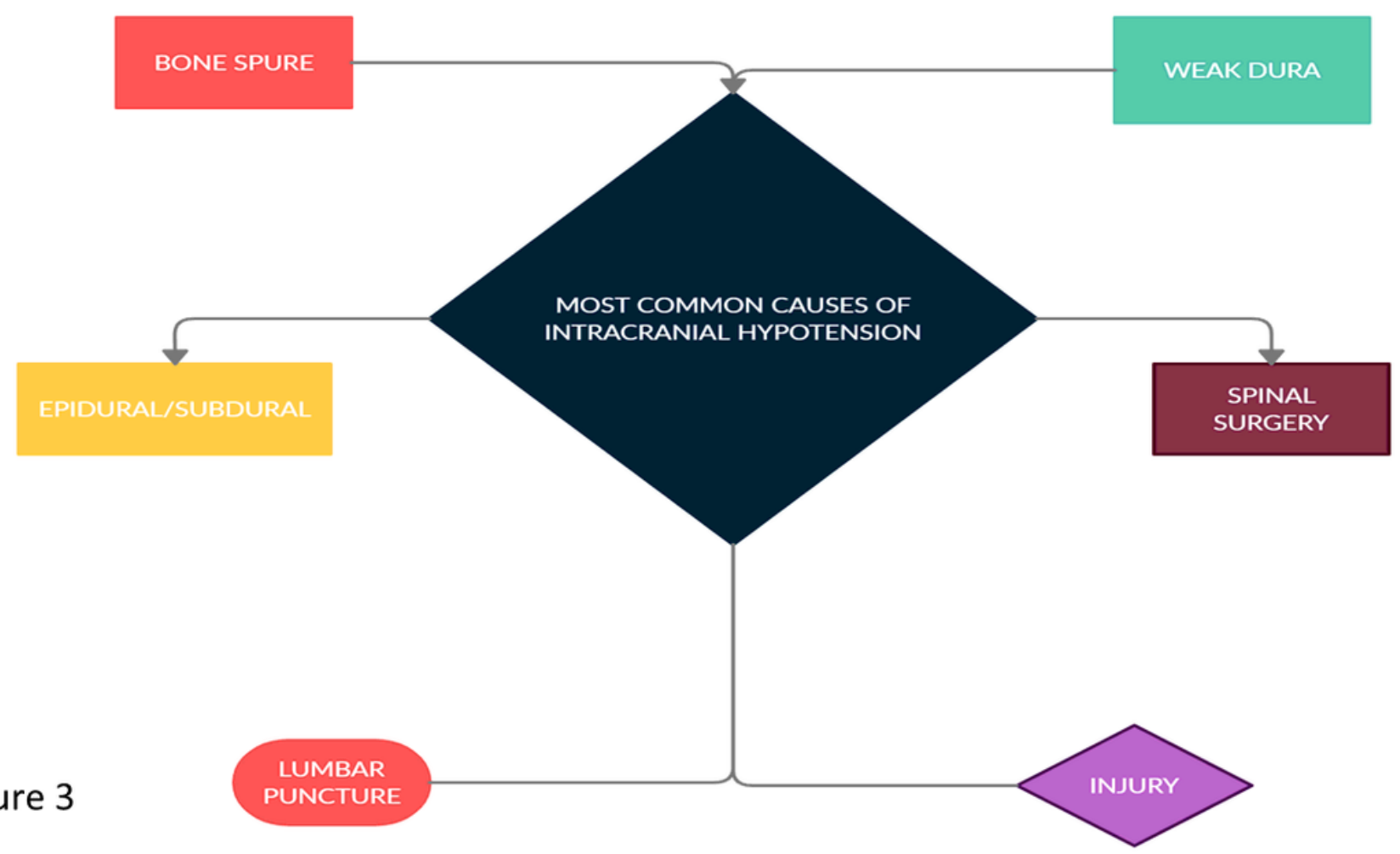

Figure 3

Diagram, including the most typical cause of $\mathrm{SIH}$.

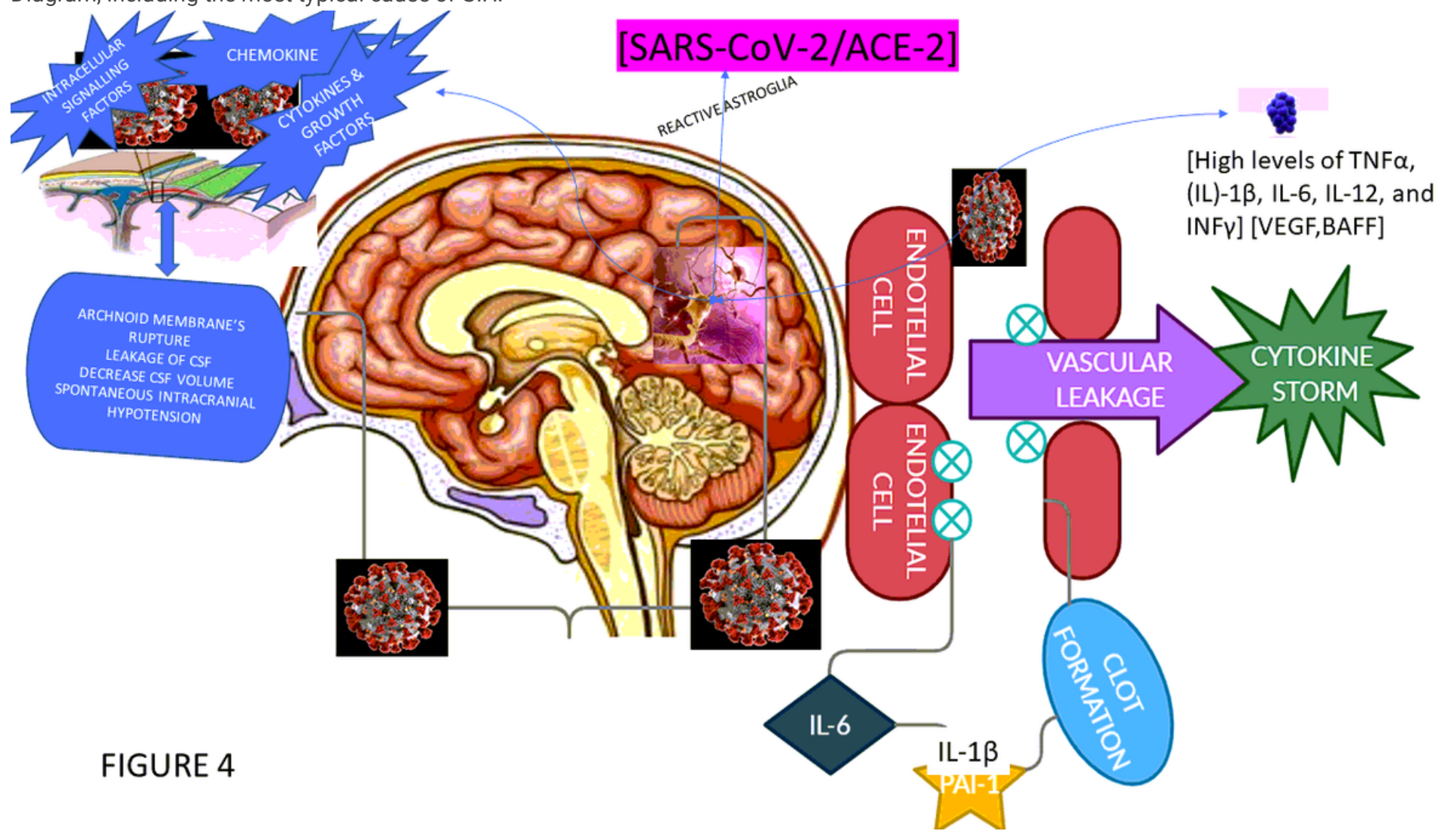


Propose a SIH mechanism in patients presenting severe COVID-19 from reactive astrocytes and cytokine storm.

Page 16/16 